\title{
Body weight changes in early onset Narcolepsy: implying compensatory mechanisms
}

Zongwen Wang, MD1; Huijuan Wu, MD, PhD*

To the Editor

We thank Dr Kovalská et al. for their special interest in our paper, and we thank the editors for giving us the opportunity to interpret our work. The findings of Dr Kovalská contribute to the understanding of the long-term status and outcome of patients with narcolepsy with cataplexy [1]. Their observations on characteristics of BMI were consistent with our findings in body mass index (BMI) [2]. However, they may have misunderstood our findings on BMI and BMI growth [3]. Our data showed significant interaction in time and BMI growth, but not in time and BMI, between patients and controls. Moreover, because of the methodological differences, their patients aged over 60 years and the interval between age at onset and diagnosis was 13.1 years $( \pm 14.8)$, the findings of Dr Kovalská have significant limitations, such as comorbid disease and nutritional problems.

With low incidence and difficulties in identify narcolepsy, the diagnosis is often delayed for decades [4-6]. It is a challenge to analyze the disease evolution of early onset cases in early stage (within three to four years). The incidence of narcolepsy in China has increased significantly due to $2009 \mathrm{H} 1 \mathrm{N1}$ winter influenza pandemic [7, 8]; this enabled us to explore the clinical features of early onset cases of narcolepsy. Therefore, our study mainly focused on the changes of clinical characteristics in the early stage of disease onset, as well as long-term follow-up.

According to our clinical observations, we noticed that the severity of partial symptoms was reduced (such as excess daytime sleepiness (ESS), 24h sleep time (24ST), and the severity of cataplexy) with the evolution of the time in both patients 
with drugs (118 patients, aged 13.68 \pm 3.32 years) and drug naïve (14 patients, aged $14.17 \pm 2.55$ years). For the same groups of patients, the objective findings of multiple sleep latency tests (MSLT)--including multiple sleep latency and sleep onset rapid eye movement periods (SOREMPs)--did not change significantly (Table 1). The severity of cataplexy is assessed by the revised cataplexy severity rating score (Table 2).

Our findings are consistent with Dr. Pizza et al.'s work, Clinical and polysomnographic course of childhood narcolepsy with cataplexy [9]. They reported that onset of childhood narcolepsy with cataplexy is characterized by an abrupt increase of 24ST, generalized hypotonia and active movements. With time, the presentation of cataplexy evolves into the classic one, whereas 24ST decreases, returning to moderate levels. They concluded that narcolepsy with cataplexy is not a stable phenotype in children, but displays a characteristic evolution with an abrupt onset followed by an improvement over time. Our findings add new data to literature and reveal crucial compensatory mechanisms that underlie the remission of narcolepsy symptoms.

We therefore conclude that persistently increased BMI reveals the lifelong body characteristics of narcolepsy. The gradual restoration of BMI growth rate, together with partial relief of clinical symptoms, eg, 24ST, ESS and the severity of cataplexy present the remission trajectory of symptoms with time of the disease. The significantly lower basal metabolic rate (BMR) in early stage and gradually increased BMR contribute to changes in growth rate of BMI [2]. Our study shows important compensatory mechanisms in metabolic disorder in early onset NC patients, but further research is required to reveal the complex mechanisms of evolution on sleep and cataplexy in narcolepsy. 
Table 1 ESS, Sleep time, and MSLT data of patients with NC at baseline and at follow-up evaluations.

\begin{tabular}{lccc}
\hline \multirow{2}{*}{ Patients group } & $\begin{array}{c}\text { Baseline } \\
\text { (within one year after } \\
\text { disease onset) }\end{array}$ & \multicolumn{2}{c}{ Follow up (3-5 years after disease onset) } \\
\cline { 3 - 4 } & $617.22 \pm 67.87$ & with drugs & drug naïve \\
\hline MST & $3.04 \pm 0.46$ & $3.12 \pm 1.43$ & $517.48 \pm 93.47^{*}$ \\
SSLT-sl & $3.66 \pm 0.57$ & $3.54 \pm 0.67$ & $2.98 \pm 1.43$ \\
EoREMPs & $16.47 \pm 2.19$ & $12.58 \pm 2.44^{*}$ & $3.29 \pm 0.48$ \\
ESS-score & $4.24 \pm 1.02$ & $1.64 \pm 0.53^{* *}$ & $13.14 \pm 1.95^{*}$ \\
Cataplexy score & &
\end{tabular}

Abbreviations: 24ST, 24h sleep time; ESS, Epworth Sleepiness Scale; MSLT, multiple sleep latency test; sl, sleep latency; SOREMPs, sleep onset rapid eye movement periods; drugs treatment involved venlafaxine (75-150mg/d) and/or methylphenidate $(18-36 \mathrm{mg} / \mathrm{d})$. ${ }^{*} \mathrm{p}<0.05,{ }^{* *} \mathrm{p}<0.01$, compared with baseline by t-test.

Table 2 Revised Cataplexy Severity Rating Score [10]

Severity Score Description of cataplexy attack

0

No cataplexy attack (The frequency were calculated within the latest a month)

1

Partial attacks involved weakness of head, neck and upper limbs, eg, head drop, jaw opening or dysarthria; regardless the frequency.

Partial attacks involved weakness in the trunk and limbs, but the patients can

maintain posture without collapse; regardless the frequency.

Complete muscular weakness, patients may slump to the ground; the frequency is occasional, less than once a week.

4

Frequently complete muscular weakness; the frequency is more than once a week, less than once a day. 


\section{Acknowledgment}

This study was supported by research grants from the National Science Foundation of China $(81100990,81171252)$ and the Ministry of Science and Technology Plan Fund Major Projects (2011ZXJ09202-015).

\section{References}

[1] Kovalská P, Kemlink D, Nevšímalová S, et al. Narcolepsy with cataplexy in patients aged over 60 years: a case-control study. Sleep Med. 2016;26:79-84.

[2] Wang Z, Wu H, Stone WS, et al. Body weight and basal metabolic rate in childhood narcolepsy: a longitudinal study. Sleep Med. 2016;25:139-44.

[3] Kovalská P, Kemlink D, Topinková E, et al. Higher body mass index in narcolepsy with cataplexy: lifelong experience. Sleep Med. 2016. http://dx.doi.org/10.1016/j.sleep.2016.11.010

[4] Overeem S, Mignot E, GertvanDijk J, et al. Narcolepsy: clinical features, new pathophysiologic insights, and future perspectives. J CLIN NEUROPHYSIOL. 2001;18:78-105.

[5] Morrish E, King MA, Smith IE, et al. Factors associated with a delay in the diagnosis of narcolepsy. Sleep Med. 2004;5:37-41.

[6] Thorpy MJ, Krieger AC. Delayed diagnosis of narcolepsy: characterization and impact. Sleep Med. 2014;15:502-7.

[7] Han F, Lin L, Warby SC, et al. Narcolepsy onset is seasonal and increased following the 2009 H1N1 pandemic in China. Ann Neurol. 2011;70:410-7.

[8] Wu H, Zhuang J, Stone WS, et al. Symptoms and occurrences of narcolepsy: a retrospective study of 162 patients during a 10-year period in Eastern China. Sleep Med. 2014;15:607-13.

[9] Pizza F, Franceschini C, Peltola $\mathrm{H}$, et al. Clinical and polysomnographic course of childhood narcolepsy with cataplexy. Brain. 2013;136:3787-95.

[10] Murali $\mathrm{H}$, Kotagal S. Off-label treatment of severe childhood narcolepsy-cataplexy with sodium oxybate. Sleep. 2006;29:1025-9.

Zongwen Wang, MD

Department of Neurology, Changzheng Hospital, Second Military Medical University, Shanghai, China Huijuan $\mathrm{Wu}, \mathrm{MD}, \mathrm{PhD} *$

Department of Neurology, Changzheng Hospital, Second Military Medical University, Shanghai, China The Center for sleep sciences and health, Institute of Sociology, Shanghai academy of social sciences Shanghai, China

* Corresponding author.

E-mail address: huijuan.w@163.com (Huijuan Wu) 